\title{
Study on Real-Time Simulation Analysis and Inverse Analysis System for Temperature and Stress of Concrete Dam
}

\author{
Lei Zhang, ${ }^{1}$ Yi Liu, ${ }^{1}$ Bingqi Li, ${ }^{1}$ Guoxin Zhang, ${ }^{1}$ and Songtao Zhang ${ }^{2}$ \\ ${ }^{1}$ State Key Laboratory of Simulation and Regulation of Water Cycle in River Basin, \\ China Institute of Water Resources and Hydropower Research, Beijing 100038, China \\ ${ }^{2}$ The Lower Reaches of the Haihe River Management Bureau, HWCC, MWR, Tianjin 300061, China \\ Correspondence should be addressed to Lei Zhang; zhangl@iwhr.com
}

Received 11 June 2014; Revised 14 July 2014; Accepted 18 July 2014

Academic Editor: Song Cen

Copyright (C) 2015 Lei Zhang et al. This is an open access article distributed under the Creative Commons Attribution License, which permits unrestricted use, distribution, and reproduction in any medium, provided the original work is properly cited.

\begin{abstract}
In the concrete dam construction, it is very necessary to strengthen the real-time monitoring and scientific management of concrete temperature control. This paper constructs the analysis and inverse analysis system of temperature stress simulation, which is based on various useful data collected in real time in the process of concrete construction. The system can produce automatically data file of temperature and stress calculation and then achieve the remote real-time simulation calculation of temperature stress by using high performance computing techniques, so the inverse analysis can be carried out based on a basis of monitoring data in the database; it fulfills the automatic feedback calculation according to the error requirement and generates the corresponding curve and chart after the automatic processing and analysis of corresponding results. The system realizes the automation and intellectualization of complex data analysis and preparation work in simulation process and complex data adjustment in the inverse analysis process, which can facilitate the real-time tracking simulation and feedback analysis of concrete temperature stress in construction process and enable you to discover problems timely, take measures timely, and adjust construction scheme and can well instruct you how to ensure project quality.
\end{abstract}

\section{Introduction}

Water conservancy and hydraulic power projects are getting bigger and bigger; diverse construction means are adopted and construction speed is high. However, concrete cracks often occur during construction, which produced the saying "no dam can be free from crack." At home and abroad, many dams suffer from serious cracks during construction period or operation period; completions of some dams are delayed for several years and consequently reservoir filling and power generation have to be delayed. Due to cracks, safety and normal operation of some dams are severely affected, and some dams even become wasted. Most of dam crack accidents are caused by thermal stress, especially the cracks which appeared in the process of construction.

Up to now, dam cracks occurring during construction and early operation are settled mainly by controlling temperature during construction. So, during concrete dam construction, it is very necessary to enhance the real-time monitoring and scientific management and analysis of concrete temperature. In order to ensure construction quality, tracking and analyzing temperature course and stress changes of concrete dam during construction in real time, and timely learning about the current construction conditions and predicting future conditions, can enable you to discover problems timely, take measures timely, and adjust construction scheme and can well instruct you how to ensure project quality. In earlier years, because the simulation calculation technique was backward, construction process simulation was very complicated and so forth; it was very hard to realize real-time thermal stress simulation analysis and inverse analysis. As the computer and internet technologies develop, the database technology is applied, and some breakthroughs are made in construction simulation calculation methods and so forth; real-time thermal stress simulation analysis and inverse analysis are not infeasible any more. 


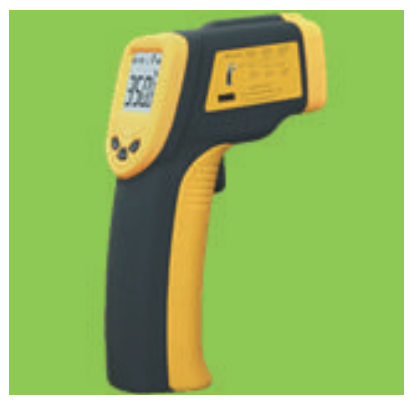

(a) Infrared thermometer

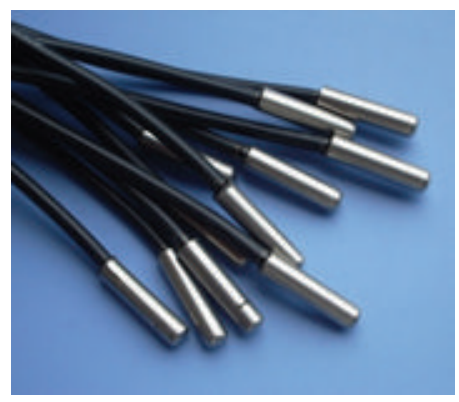

(b) Digital temperature sensor

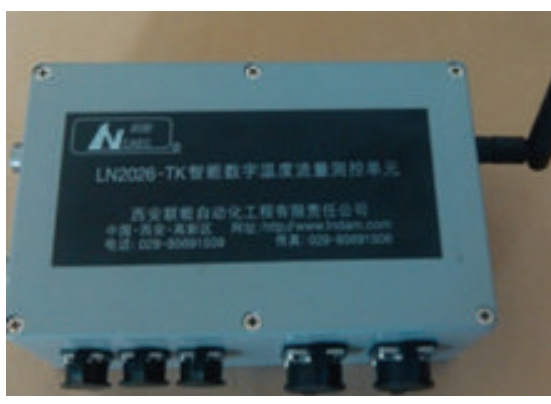

(c) Temperature recorder

FIgURE 1: Automatic measurement equipment.

\section{Design of Technical Solution of the System}

This paper proposes a temperature and stress real-time simulation analysis and inverse analysis system based on database.

For the data information in the database, there is a module to realize information acquisition. In this module, automatic measurement equipment is used to collect data automatically or semiautomatically. These data include mixing temperature, forming temperature, placing temperature of concrete, concrete temperature after placement, flow rate in the pipe, water temperature in the pipe, temperature gradient on the dam surface, air temperature, solar radiation heat, horizontal displacement of dam, and vertical displacement of dam. The monitoring equipment automatically collects concrete temperature after placement, flow rate, water temperature, temperature gradient on the dam surface, air temperature, solar radiation heat, and so forth, and also other basic information for the simulation analysis can be obtained semiautomatically or by manual work. Figure 1 shows some measurement equipment.

Technical scheme of the temperature and stress realtime simulation analysis and inverse analysis system is to utilize the database technique, high performance computing technology, the web programming technology, and other technologies to build the data acquisition and analysis module which is used to acquire available data from the database in real time and process and analyze data to produce calculating data documents, to further build the HPC (high performance computing) simulation module, and to calculate thermal stresses based on the calculation platforms of various architectures by calling SapTis [1] simulation calculation software and then to build the smart inverse analysis module for feedback analysis on temperatures and figuring out real temperatures and stresses by further calculation, and at last to build the postprocessing module to visually show calculation results and give safety prewarning as per the safety factor.

The whole system consists of four main subsystems, namely, data acquisition and analysis subsystem, HPC simulation subsystem, intelligent inverse analysis subsystem, and postprocessing analysis and prewarning subsystem. Each subsystem is a module, and the subsystems constitute the whole real-time thermal stress simulation analysis and inverse analysis system. The system is featured by real time,

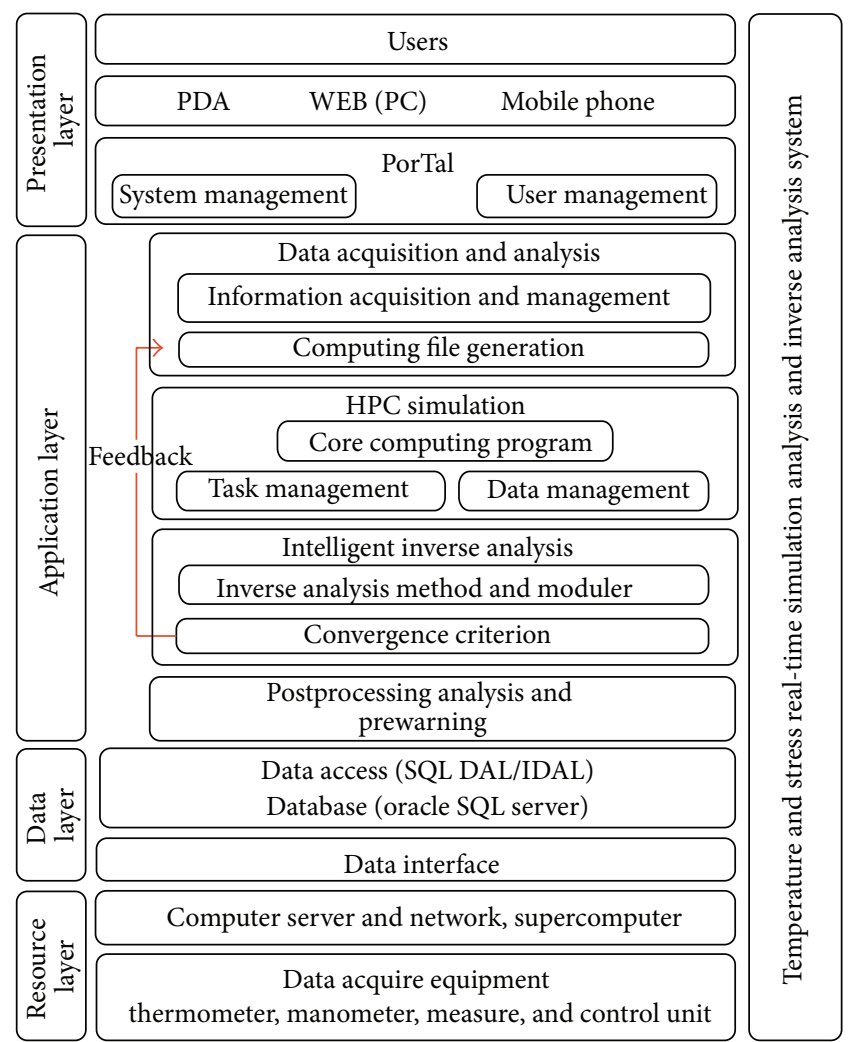

Figure 2: Architecture of the system.

automation, intelligence, and visualization. It can realize the real-time, smart, and quick analysis and inverse analysis of construction quality of concrete placement site, and visual presentation of analysis results and safety prewarning.

\section{System Structure}

Figure 2 is the architecture of the system [2,3]. Figure 3 is the module and workflow of the system. The whole system consists of the four subsystems: the data acquisition and analysis subsystem, the HPC simulation subsystem, the intelligent inverse analysis subsystem, and the postprocessing analysis and prewarning subsystem. And there is also a 


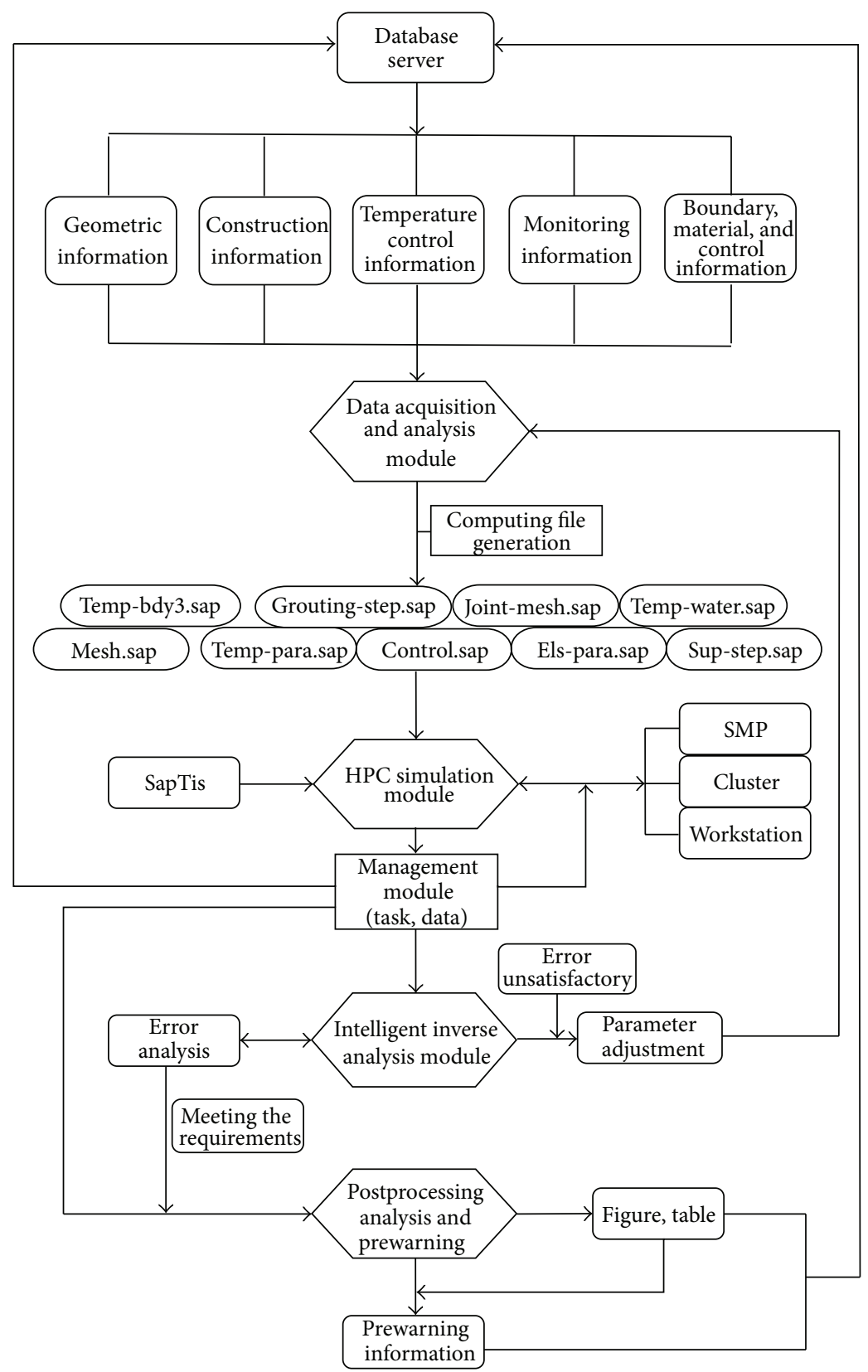

FIgURE 3: Module and workflow of the system.

small management module between HPC simulation module and intelligent inverse analysis module; this module could be considered to belong to the intelligent inverse analysis subsystem.

The system is of 4-layer B/S architecture. The first layer, presentation layer: it is the data acquisition layer in the layout of a webpage showing the system administration interface, managing data, showing results by chart, showing prewarnings, and so forth. The presentation layer separates the system operation interface from the system function realization. The second layer, application layer: this layer includes WEB server, application server, and middleware technology realization. It is the service logic realization layer of the application system, is the core of the system, receives function requests from the presentation layer, and consists of logic entity realizing all the service functions, and these logic entities embody database trigger, storage procedures, and various functional modules, like SapTis computation module, inverse analysis control module, results manipulation module, and so forth. The third layer, data layer: this layer stores and manages various information based on SQLserver database, realizes access to various databases and data sources, and is also the only interface for the system to visit other data sources. The fourth layer, resource layer: mainly some hardware includes computer server and network equipment and supercomputer for data management 
and FEM simulation. In addition to this, there are also some data that acquire equipment of temperature control information. The B/S-based four-layer system architecture distributes the presentation layer, application logic, and data source layer to different units, to enhance the expansibility of the system, enable the system to support more clients and dynamically allocate WEB server and application server as per page views, enhance the expansibility, safety, and code reusability of the system, and simplify maintenance of the system.

\section{Detailed Design of Each Subsystem}

4.1. Data Acquisition and Analysis Subsystem. The data acquisition and analysis subsystem acquires available data mainly through the database technique [4], automatically processes and analyzes all acquired data, and produces data documents required by SapTis simulation calculation. The main data documents and realization methods for these main data documents are shown below.

4.1.1. Mesh Information Document: Mesh.sap. It mainly includes some information such as elements, nodes, loads, boundary, and concrete layer.

Realization Methods. Select concrete placement cabin and determine cabin number to form mesh.sap document through the system, which includes computing mesh data, boundary conditions, and initial condition information required for simulation computing, because generation of mesh.sap needs a series of processing works, which realize the formation of mesh.sap file through batched processing.

4.1.2. Concrete Placement Information Document: Supstep.sap. It includes information such as concrete placement layer and time-steps.

Realization Methods. After selecting concrete placement cabin, the system exports the placement time and placement cabin elevation. Access mesh.sap to conduct segmentation of the whole computing time process over each placement cabin via placement time, and the placement time of each cabin is a starting point with which to conduct standardized segmentation to satisfy the temperature field calculation requirements, and the segmentation process can adopt required formula method in accordance with the accuracy of the temperature calculation.

4.1.3. Material Parameter Information Document: Elspara.sap. It includes information such as linear expansion coefficients, deformation modulus, Poisson's ratio, volume weight, creep, self-grown volume deformation, and $\mathrm{MgO}$.

Realization Methods. Parameter information of various materials is stored in the system. After selecting computing domain, the system generates files of parameter information of various materials based on each calculation domain.
4.1.4. Cooling Information Document: Temp-para.sap. It includes thermal parameters such as the heat of hydration process, specific heat, thermal conductivity coefficient, and cooling parameter of each period of the water pipe.

Realization Methods. Obtain actual cooling information and planned placement information of each cabin from the system to automatically generate cooling information file. The cooling periods are limited to 10 . Each cabin is equipped with a pipe information table, which is obtained by conducting simple calculation over some cooling parameters and starting and ending time of each placement cabin's cooling periods.

4.1.5. Boundary Condition Information Document: Tempbdy3.sap. It includes boundary conditions such as air temperature, water temperature, water level, and sand level of various calculation time-steps.

Realization Methods. The system can export files needed through embedding auxiliary program based on air temperature information and water temperature information to realize generation of this file.

4.1.6. Water Temperature Document: Temp-water.sap. This file is generated through accessing information of the water temperature in the database.

4.1.7. Joint Element Information Document: Joint-mesh.sap. This file includes information such as constitutive model of the joint and node and joint element.

Realization Methods. Through embedding joint element searching program, the system generates joint-mesh.sap file of the required format.

4.1.8. Grouting Information Document: Grouting-step.sap. The system can realize generation of the file in accordance with the information in "sup-step.sap" file and grouting progress through embedding program to search corresponding pouring steps.

4.1.9. Other Computing Auxiliary Information Control Documents: Control.sap. It can be generated through accessing the data setting in the database.

Meanwhile, in order to coordinate operation of inverse analysis subsystem, the data acquisition and analysis subsystem can provide data files required by the inverse analysis subsystem.

4.2. HPC Simulation Subsystem. The system conducts temperature and stress calculation through calling SapTis simulation software in various computing platforms of different system structures. SapTis is a large-scale linear and nonlinear simulation and analysis program for structural temperature field and stress field and has a multifunction such as concrete pouring, hole room excavation, temperature, and seepage, deformation and stress field simulation, and structural nonlinear analysis. For the function of thermal stress simulation, 


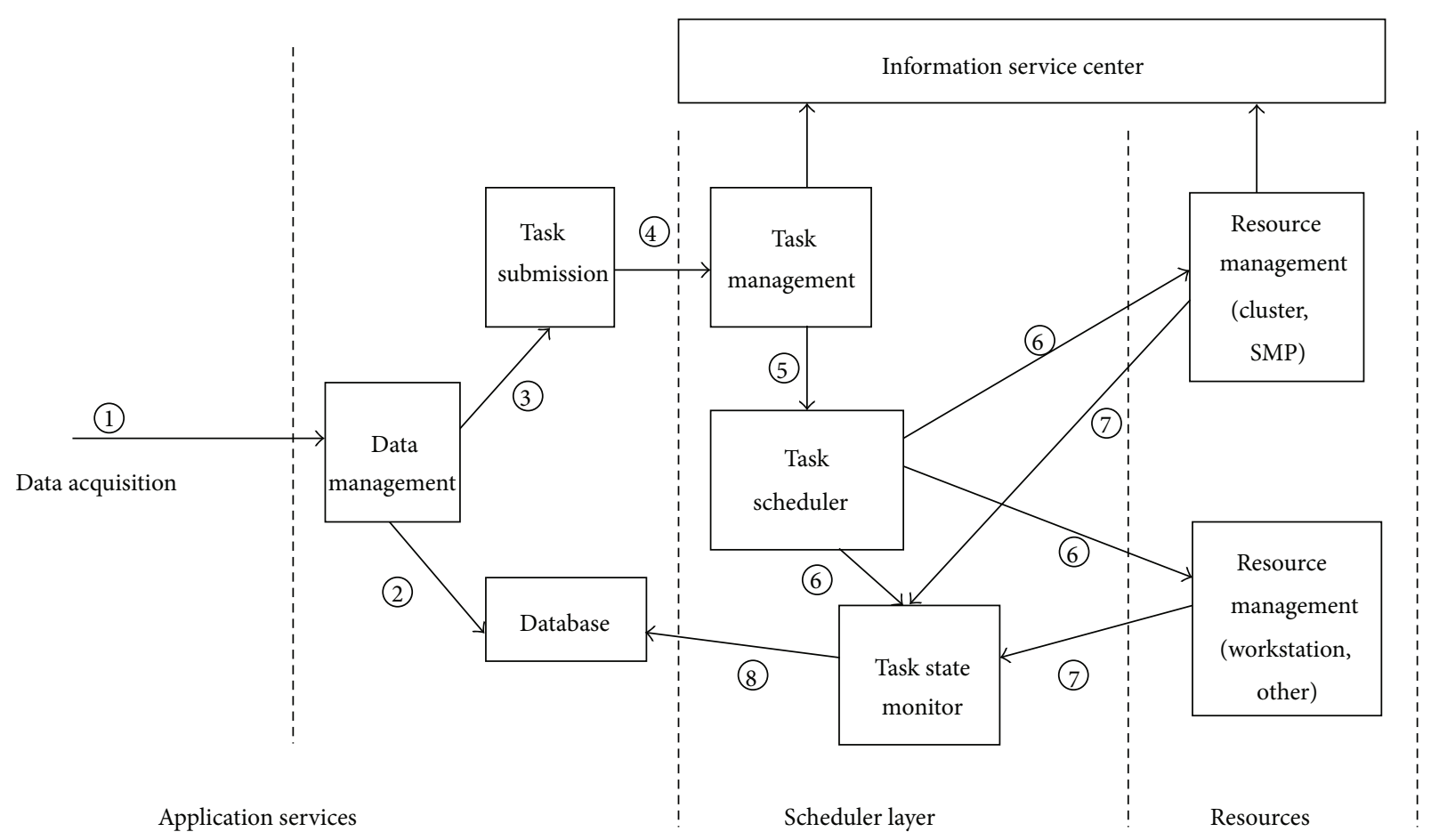

FIGURE 4: Task submitting process of the HPC simulation subsystem.

it has some models $[5,6]$ such as hydration models, water cooling models, basic thermal model, creep models, and autogenous deformation and $\mathrm{MgO}$ linear expansion models. Besides that, SapTis provides abundant solvers for the linear algebraic equations of FEM.

The system has integrated SapTis simulation software. Prior to operation, the subsystem will firstly pack 9 data files and SapTis program formed in the data acquisition and analysis subsystem and submit this program package to the task scheduler as a computing task, and then the scheduler submits the task to the hardware resource platform; the program package can be automatically decompressed and the hardware resource platform can be a single server or a workstation and can also be a cluster or multicore parallel machine $[6,7]$ in accordance with need. After submission of the tasks, SapTis program can adopt either serial or parallel computing model, which will be determined through the data management interface of the presentation layer. In addition, the management module can transmit the calculation results to the intelligent inverse analysis module or store the data in the database. Figure 4 shows the task submitting process of the HPC simulation subsystem, and the number shows the sequence of the workflow.

4.3. Intelligent Inverse Analysis Subsystem. This subsystem automatically analyzes and feeds back current concrete temperature and adjusts associated parameters as per set inverse analysis temperature errors, until the error requirement is met. The inverse analysis error is determined based on the concrete cooling process and historical critical moments, and each cabin of concrete takes the actual measured temperature at the three points as the basis: one is in the middle of the cabin and the other is near the upstream and downstream of the cabin. There is one temperature measurement value every day. According to the turning points of temperature process, the cooling process falls into the phase I cooling temperature rising stage, the phase I cooling temperature dropping stage, the phase I temperature controlling stage, the medium phase cooling temperature dropping stage, the medium phase cooling temperature controlling stage, the phase II cooling temperature dropping stage, and the phase II cooling temperature control stage. The historical critical moments refer to the turning point moments in the process of temperature measurement, including the highest temperature occurrence moment, the phase I cooling ending moment, the medium phase cooling starting moment, the medium phase cooling end moment, the phase II cooling starting moment, the phase II ending moment, and moments of other turning points of the temperature process. The average error of each cooling phase is $\pm 0.5^{\circ} \mathrm{C}$, and the temperature error of each historical critical moment is $\pm 0.5^{\circ} \mathrm{C}$. Each feedback calculation can come to an end by simultaneously meeting the requirements of both errors. If the errors cannot satisfy the requirements, the system can automatically adjust the related parameters such as cooling water temperature, cooling water flow, and cooling time by empirical model until all the temperature errors of each cabin meet the requirements.

Figure 5 shows a standard temperature curve with only the first phase cooling and medium phase cooling. The feedback analysis subsystem will determine whether the temperature control parameters should be adjusted in accordance with errors of each simulation computing. In the figure, $T_{m}$ 
represents the highest temperature, $T_{c 1}$ is target temperature of the first cooling phase, $T_{c 2}$ is target temperature of the medium cooling phase, $D 1$ is the moment of reaching the highest temperature moment, $D 2$ is the moment of reaching the target temperature of the first cooling phase, D3 is the moment of reaching the target temperature of medium cooling phase, and D4 is the moment of ending temperature control. Calculation method of errors of each cooling phase is shown in (1) to (4). $r_{f k}, r_{f j}, r_{m j}$, and $r_{m k}$, respectively, represent the first phase temperature control error, the first phase temperature drop error, medium phase temperature drop error, and medium phase temperature control error. $T_{i c}$ and $T_{i m}$, respectively, represent calculated temperature and measured temperatures every day. Error of each critical moment is shown in (5) to (8). $r_{m}, r_{1 c}, r_{c 2}$, and $r_{f c 2}$, respectively, represent error of $D 1, D 2, D 3$, and $D 4$ moments. $T_{m c}, T_{c 1 c}, T_{c 2 c}$, and $T_{f c 2 c}$, respectively, represent the highest calculated temperature, the target cooling temperature of the first calculation phase, the target cooling temperature of the medium phase of calculation, and the calculation target cooling temperature of controlling temperature ending moment in medium cooling phase.

$T_{m m}, T_{c 1 m}, T_{c 2 m}$, and $T_{f c 2 m}$, respectively, represent the highest measured temperature, the measured target cooling temperature of the first phase, the measured target cooling temperature of the medium phase, and the measured target cooling temperature of controlling temperature ending moment in medium cooling phase.

Each feedback calculation will comprehensively take into account positive and negative value of errors of the critical moments and the process of each cooling phase and then adjust cooling parameters of corresponding positions so as to gradually achieve convergence of the feedback calculation:

$$
\begin{gathered}
r_{f k}=\frac{\sum_{i=1}^{D 1}\left(T_{i c}-T_{i m}\right)}{D 1}, \\
r_{f j}=\frac{\sum_{i=D 1}^{D 2}\left(T_{i c}-T_{i m}\right)}{D 2-D 1}, \\
r_{m j}=\frac{\sum_{i=D 2}^{D 3}\left(T_{i c}-T_{i m}\right)}{D 3-D 2}, \\
r_{m k}=\frac{\sum_{i=D 3}^{D 4}\left(T_{i c}-T_{i m}\right)}{D 4-D 3}, \\
r_{m}=T_{m c}-T_{m m}, \\
r_{c 1}=T_{c 1 c}-T_{c 1 m}, \\
r_{c 2}=T_{c 2 c}-T_{c 2 m}, \\
r_{f c 2}=T_{f c 2 c}-T_{f c 2 m} .
\end{gathered}
$$

4.4. Postprocessing Analysis and Early Warning Subsystem. This module read calculation results from the database, analyzes and processes the result data, shows calculation

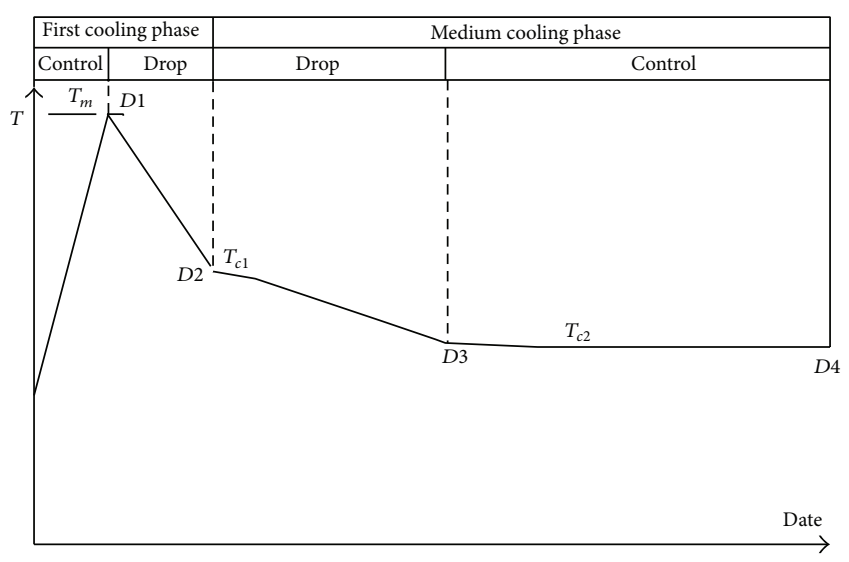

Description:

$T_{m}:$ maximum temperature limit

$T_{c 1}$ : the first cooling phase target temperature

$T_{c 2}$ : the medium cooling phase target temperature

FIGURE 5: Standard temperature process curve.

results visually, and gives safety prewarning as per the safety coefficient value. The postprocessing and analysis function realizes visualized data output through the embedded postprocessing program, realizes the automatic generation of postprocessing diagrams by batch processing commands, and realizes display in the system by the web embedded photo module: including temperature change process curve, stress change process curve, safety coefficient change process curve, maximum and minimum temperature contour lines diagram and cloud charts, maximum and minimum stress contour line diagram and cloud charts, safety coefficient contour line diagram and cloud charts, and typical position temperature and stress comparative table. Figure 6 shows some display of the results. The other function realizes the smart safety coefficient analysis of each position. It compares the safety coefficient of each position with the allowed safety coefficient set and outputs the coordinates of the position where the safety coefficient is below the standard and gives a prewarning on the presentation layer.

\section{Conclusions}

The system can be used in construction of concrete dam and other concrete works, to enhance real-time supervision and scientific management over concrete temperature [8]. The system can trace and analyze the temperature change process and stress changes of concrete during construction in real time, timely learn about current construction conditions, and predict future conditions. Using it you can timely discover problems, timely take actions, adjust construction schemes, and ensure construction quality. This system makes supervision over concrete construction process automatic, smart, scientific, and easy, enhances supervision efficiency of concrete construction process, and thus is well worthy of popularization. 


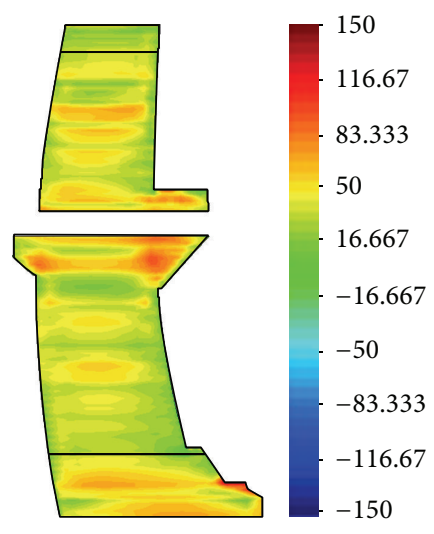

(a) Cloud drawing

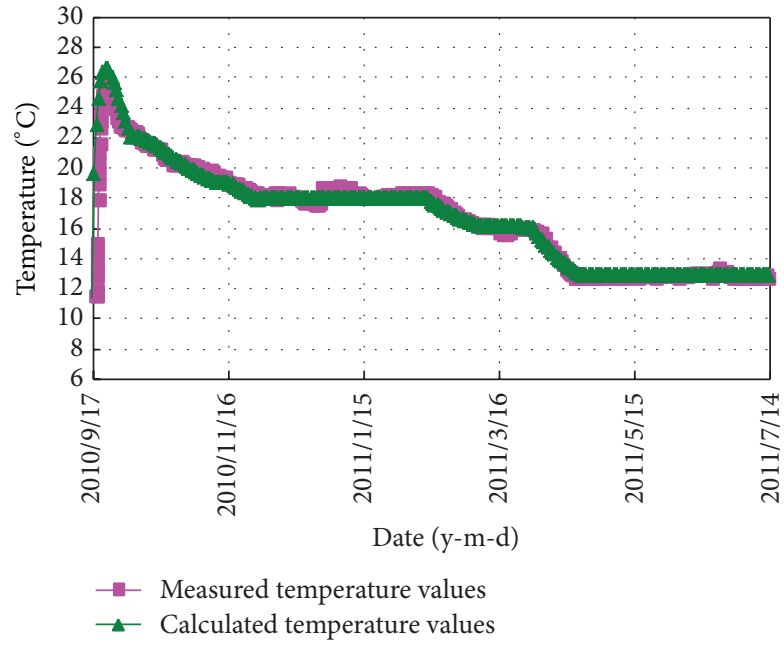

(b) Temperature process curve

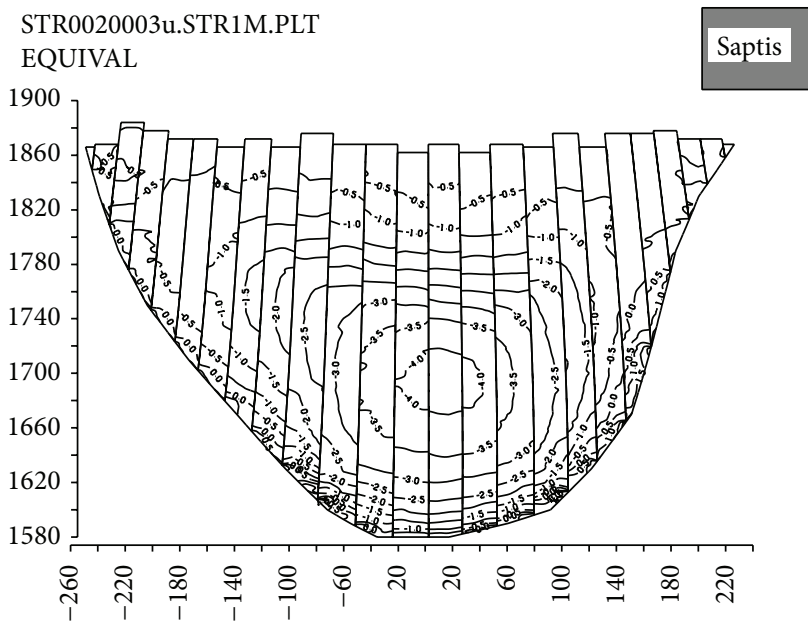

(c) Contour line diagram

FIGURE 6: Some display methods of the results.

\section{Conflict of Interests}

The authors do not have any conflict of interests with the content of the paper.

\section{Acknowledgments}

The authors would like to acknowledge the financial support of the National Natural Science Foundation of China (50909105), the National Basic Research Program (973 Programs: Grant no. 2010CB731500, Grant no. 2013CB036406, and Grant no. 2013CB035904), the National “Twelfth Five-Year” Plan for Science \& Technology Support (2013BAB06B02), the Governmental Public Industry Research Special Funds for Projects of MWR (201201050), and the Special Research Foundation of IWHR and State Key Laboratory of Simulation and Regulation of Water Cycle in River Basin.

\section{References}

[1] Z. Guoxin, "SAPTIS: development and application of multifield simulation and nonlinear analysis software(one part)," Water Resources and Hydropower Engineering, vol. 44, no. 1, pp. 31-35, 2013 (Chinese).

[2] D. Zhong, B. Cui, D. Liu, and D. Tong, "Theoretical research on construction quality real-time monitoring and system integration of core rockfill dam," Science in China E: Technological Sciences, vol. 52, no. 11, pp. 3406-3412, 2009.

[3] D. Zhong, D. Liu, and B. Cui, "Real-time compaction quality monitoring of high core rockfill dam," Science China Technological Sciences, vol. 54, no. 8, pp. 1906-1913, 2011.

[4] L. Yi, Z. Guoxin, and W. Jimin, "Digital monitoring method and system and engineering application of the construction of ultrahigh arch dam," Water Resources and Hydropower Engineering, vol. 43, no. 3, pp. 33-37, 2012 (Chinese). 
[5] Y. Q. Long, S. Cen, and Z. F. Long, Advanced Finite Element Method in Structural Engineering, Springer, Berlin, Germany; Tsinghua University Press, Beijing, China, 2009.

[6] L. Zhang, G. Zhang, L. Wang et al., "A comparative study on different parallel solvers for nonlinear analysis of complex structures," Mathematical Problems in Engineering, vol. 2013, Article ID 764237, 14 pages, 2013.

[7] L. Zhang, G. Zhang, Y. Liu, and H. Pan, "Mesh partitioning algorithm based on parallel finite element analysis and its actualization," Mathematical Problems in Engineering, vol. 2013, Article ID 751030, 6 pages, 2013.

[8] P. Lin, Q. Li, and H. Hu, "A flexible network structure for temperature monitoring of a super high arch dam," International Journal of Distributed Sensor Networks, vol. 2012, Article ID 917849, 10 pages, 2012. 


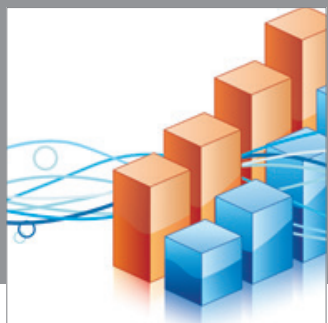

Advances in

Operations Research

mansans

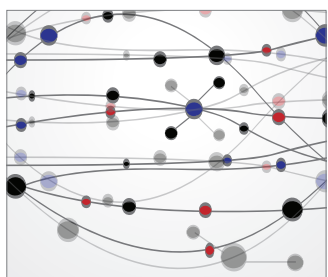

The Scientific World Journal
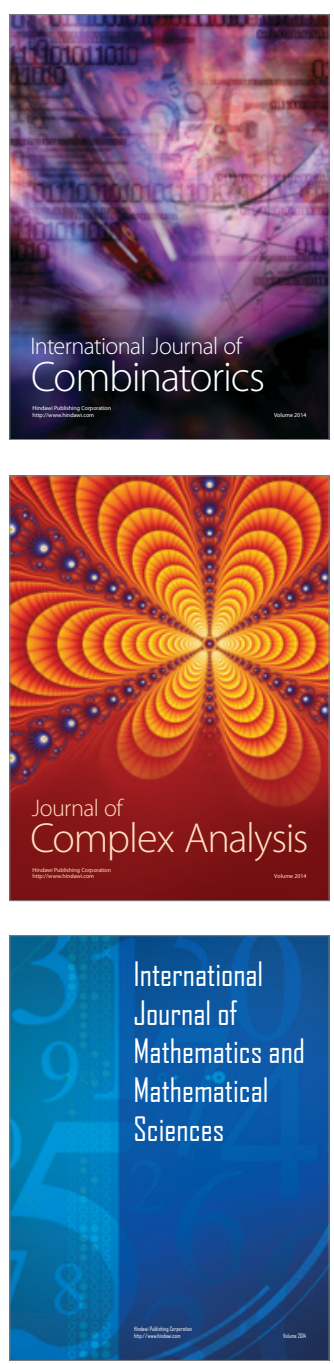
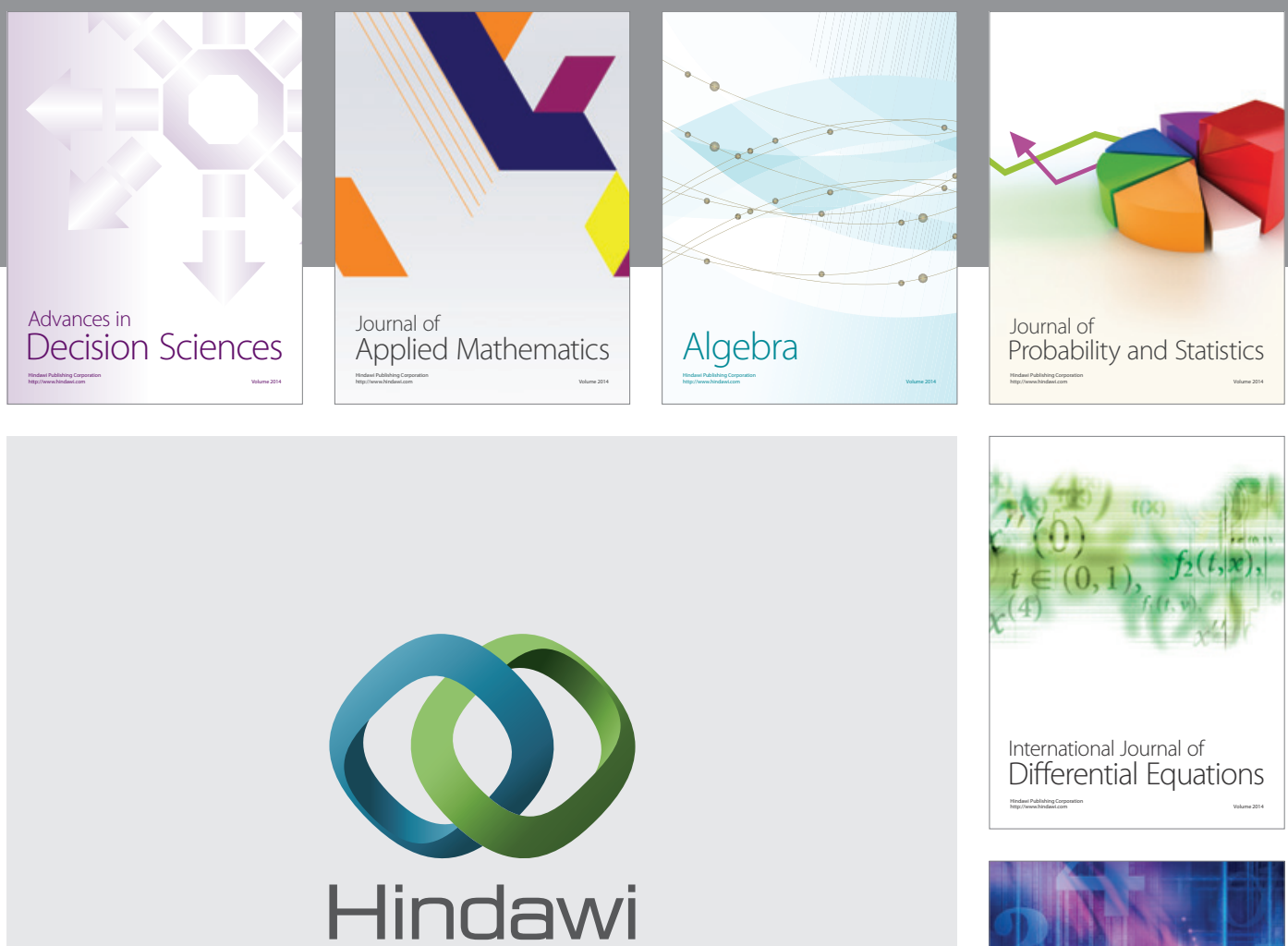

Submit your manuscripts at http://www.hindawi.com
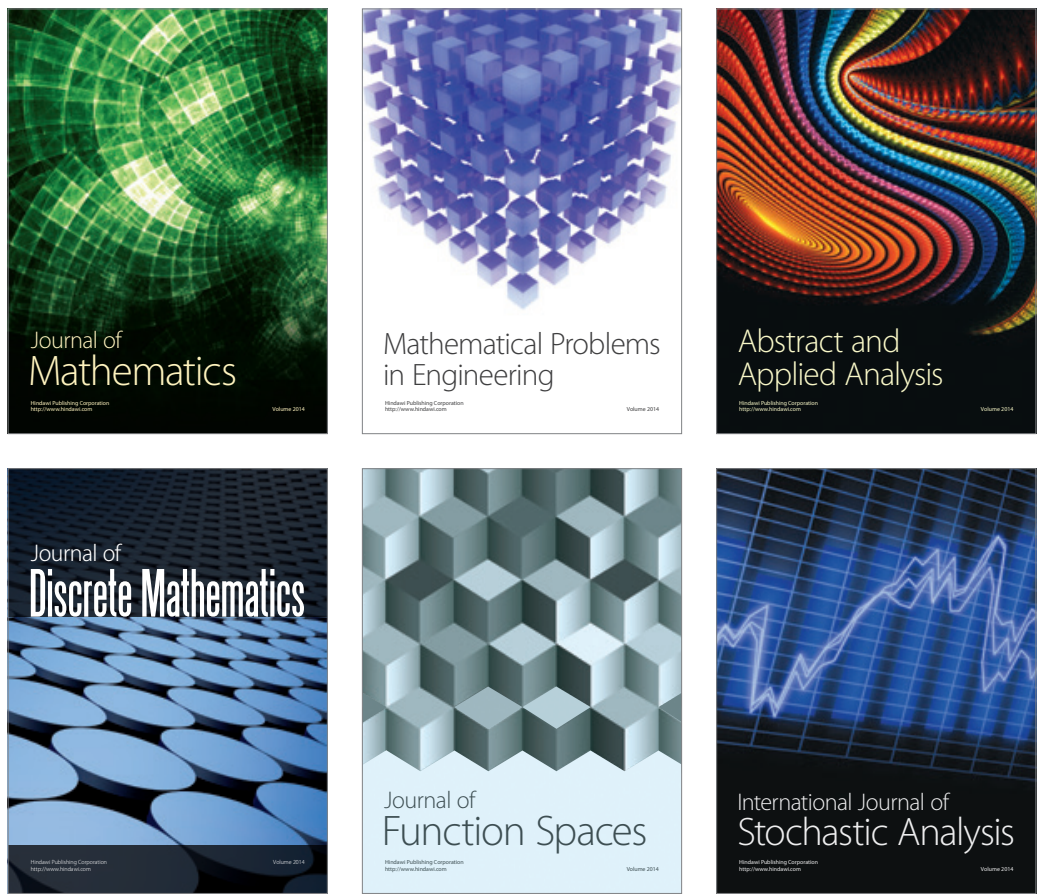

Journal of

Function Spaces

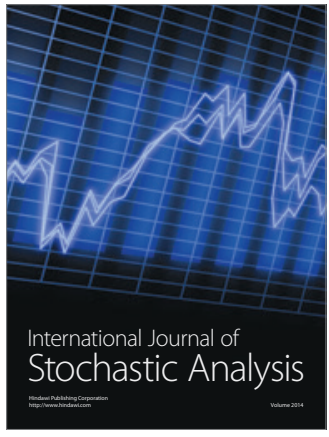

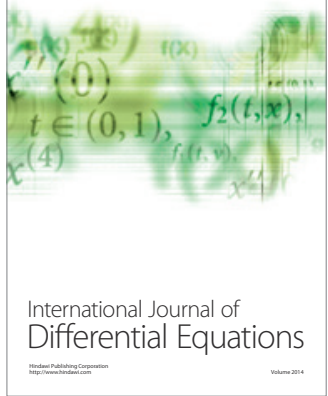
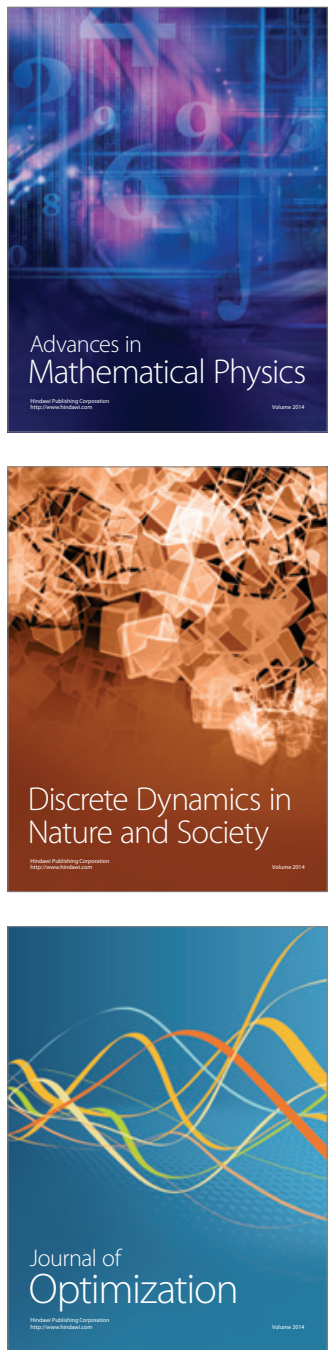